\title{
Stable Single-Layer Honeycomblike Structure of Silica
}

\author{
V. Ongun Özçelik, ${ }^{1,2}$ S. Cahangirov, ${ }^{3,4}$ and S. Ciraci, ${ }^{4, *}$ \\ ${ }^{1}$ UNAM-National Nanotechnology Research Center, Bilkent University, 06800 Ankara, Turkey \\ ${ }^{2}$ Institute of Materials Science and Nanotechnology, Bilkent University, Ankara 06800, Turkey \\ ${ }^{3}$ Nano-Bio Spectroscopy Group, Departamento Fisica de Materiales, Universidad del Pais Vasco, \\ Centro de Fisica de Materiales CSIC-UPV/EHU-MPC and DIPC, Avenida Tolosa 72, E-20018 San Sebastian, Spain \\ ${ }^{4}$ Department of Physics, Bilkent University, Ankara 06800, Turkey
}

(Received 7 October 2013; published 20 June 2014)

\begin{abstract}
Silica or $\mathrm{SiO}_{2}$, the main constituent of Earth's rocks has several 3D complex crystalline and amorphous phases, but it does not have a graphitelike layered structure in 3D. Our theoretical analysis and numerical calculations from the first principles predict a single-layer honeycomblike allotrope, $h \alpha$ silica, which can be viewed to be derived from the oxidation of silicene and it has intriguing atomic structure with reentrant bond angles in hexagons. It is a wide band gap semiconductor, which attains remarkable electromechanical properties showing geometrical changes under an external electric field. In particular, it is an auxetic metamaterial with a negative Poisson's ratio and has a high piezoelectric coefficient. While it can form stable bilayer and multilayer structures, its nanoribbons can show metallic or semiconducting behavior depending on their chirality. Coverage of dangling $\mathrm{Si}$ orbitals by foreign adatoms can attribute new functionalities to $h \alpha$ silica. In particular, $\mathrm{Si}_{2} \mathrm{O}_{5}$, where $\mathrm{Si}$ atoms are saturated by oxygen atoms from top and bottom sides alternatingly can undergo a structural transformation to make silicatene, another stable, single layer structure of silica.
\end{abstract}

DOI: 10.1103/PhysRevLett.112.246803

PACS numbers: 73.22.-f, 77.55.-g, 81.05.Zx, 81.07.-b

Trends in materials science have aimed at the discovery of single layer structures and their multilayer van der Waals composites by using advanced fabrication techniques [1]. In this endeavor, silicene, a graphenelike single layer honeycomb structure of silicon, was shown to be stable [2,3] and was later synthesized on $\operatorname{Ag}(111)$ substrate [4]. The interaction of silicene with oxygen has been a subject of active research [5-10] because it directly affects the fabrication process of silicene-based devices. Besides this, silicene-oxygen interaction leads to new two-dimensional (2D) silica crystals in different forms [5,6] and bilayer structures [11]. Recently, efforts have been devoted to grow 2D ultrathin polymorphs of silica on substrates [6,11-14], and theoretical studies have also been carried out to understand 2D silica and their defects $[9,15,16]$. In various 3D fourfold coordinated allotropes of $\mathrm{SiO}_{2}$, such as amorphous and crystalline quartz, which are commonly named as silica, $\mathrm{Si}-\mathrm{O}-\mathrm{Si}$ bonds are bent around oxygen atoms [17], except for $\beta$ cristobalite which has straight Si-O-Si bonds [18]. However, none of those allotropes of silica are known to have a graphenelike freestanding 2D structure or a graphitelike layered structure.

In this Letter, we predict a single layer allotrope of silica named $h \alpha$ silica ( $h$ denoting the single layer hexagonal lattice). Having passed through various complex stability tests, this honeycomblike structure is found to be stable. It has a more complex structure and exceptional properties as compared to those ultrathin films grown on substrates; it is a wide band gap semiconductor having a negative Poisson's ratio and high piezoelectric coefficient. The band gap can be reduced or widened depending on the direction of the applied uniaxial tensile stress. Even more remarkable, this basic structure, $h \alpha$ silica, can also form bilayer and multilayer structures and interact readily with adatoms like $\mathrm{O}, \mathrm{H}$, $\mathrm{F}$ to form a manifold of its derivatives. In particular, when $\mathrm{Si}$ atoms are saturated by oxygen, the resulting derivative $\mathrm{Si}_{2} \mathrm{O}_{5}$, is transformed to silicatene at elevated temperature. Our predictions are obtained from first-principles calculations, which are detailed in Refs. [19-26].

Silicene surface is rather reactive; $\mathrm{O}$ atoms are bonded to the bridge site with a strong binding energy of $6.17 \mathrm{eV} \mathrm{[8].}$ This bridge bonded $\mathrm{O}$ adatom can forcibly switch between two bistable equilibrium positions at either side if an energy barrier of $0.28 \mathrm{eV}$ is overcome [8]. At the transition state, the $\mathrm{Si}-\mathrm{Si}$ bond is stretched to accommodate one $\mathrm{O}$ atom near its midpoint and, hence, to form a Si-O-Si bond. Then, one may contemplate whether an exothermic process can take place, in which one $\mathrm{O}$ atom can be inserted between each $\mathrm{Si}-\mathrm{Si}$ bond of silicene concomitantly to grow a single layer honeycomblike network in $2 \mathrm{D}$ with the formula unit of $\mathrm{Si}_{2} \mathrm{O}_{3}$. Actually, a similar process occurs in 3D through the oxidation of silicon surfaces to generate amorphous $\mathrm{SiO}_{2}$.

A hexagonal unit cell including two $\mathrm{Si}$ and three $\mathrm{O}$ atoms forms a regular honeycomb network, as shown in Fig. 1(a). This structure, which we named as $h \beta$ silica in analogy to $\beta$ cristobalite in $3 \mathrm{D}$, corresponds to a shallow local minimum. When perturbed from perfect symmetry and subsequently 
(a)

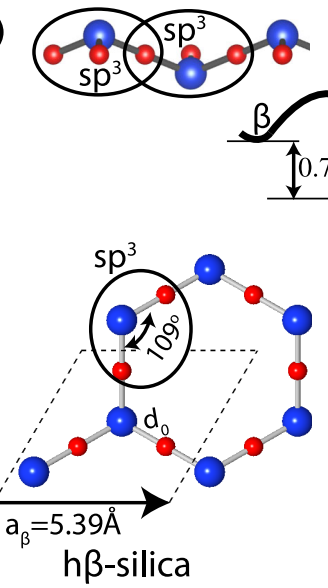

(c)

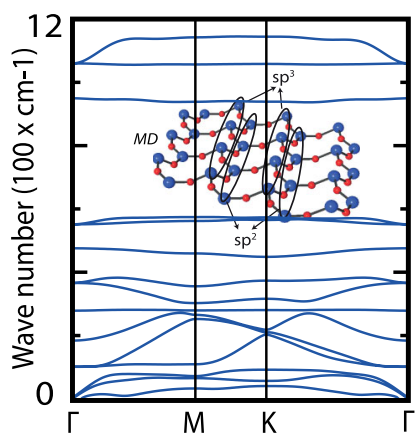

(b)
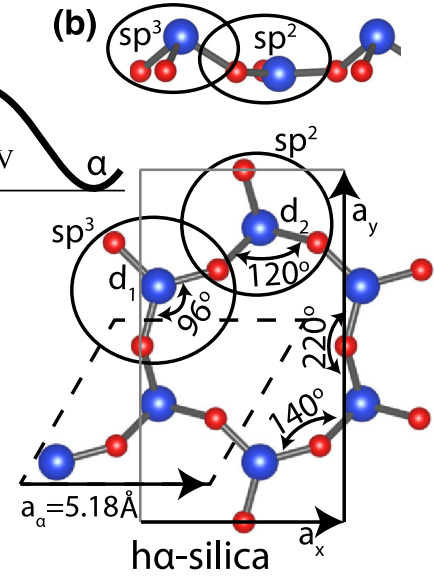

(d)

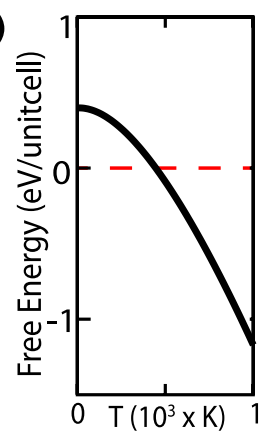

FIG. 1 (color online). (a) Side and top views of $h \beta$ silica. Large blue and small red balls stand for $\mathrm{Si}$ and $\mathrm{O}$ atoms. (b) Optimized structure of $h \alpha$ silica with its hexagonal and rectangular unit cells. Two types of $\mathrm{Si}$ atoms, i.e., $s p^{3}$ bonded and $s p^{2}$ bonded are indicated. (c) Calculated phonon bands and tilted view of the MD simulation in $4 \times 4$ unit cell. (d) Free energy versus temperature.

relaxed by the conjugate gradient method using stringent convergence criteria, $h \beta$ silica eventually transforms to a new structure, $h \alpha$ silica, and the energy is lowered by $0.7 \mathrm{eV}$. The optimized geometrical parameters of this allotrope and other physical properties are described in Fig. 1(b) and Table I. In the optimized structure, three alternating $\mathrm{Si}$ atoms of a hexagon engage in planar $s p^{2}$ bonding and the remaining three rise upward or downward [27] and form $s p^{3}$-like bonds [28]. Consequently, the rotary reflection symmetry is broken and the lattice is shrunk by $\sim 4 \%$. The straight $\mathrm{Si}-\mathrm{O}-\mathrm{Si}$ bonds are bent forming reentrant bond angles [29], such that all $\mathrm{O}$ atoms are coplanar together with the $s p^{2}$-bonded $\mathrm{Si}$ atoms. Thus, the regular hexagons are distorted where $\mathrm{Si}$ atoms are placed at the corners of hexagons, but they are buckled like silicene. Its high cohesive energy, $E_{c}=28.6 \mathrm{eV}$ per unit cell originates from mixed (covalent and ionic) bonds between $\mathrm{Si}$ and $\mathrm{O}$ atoms while its high formation energy prevents it from clustering. These rearrangements are reminiscent of the transition from ideal $\beta$ cristobalite to $\alpha$ quartz in 3D.

The rigorous answer to the question of whether the optimized structure is stable is provided by calculating the phonon frequencies with extreme accuracy. All frequencies of the infinitely large, single layer are found to be positive in the first Brillouin zone. As shown in Fig. 1(c), the calculated bands of frequencies, $\Omega(\mathbf{k})$, with a minute gap between optical and acoustical branches, clearly demonstrate that $h \alpha$ silica remains stable even if the stabilizing finite size and edge effects are absent. Otherwise, the specific eigenfrequencies of the dynamical matrix would be imaginary if any instability due to the long wavelength transversal acoustical phonons was imposed.

Although the phonon spectrum provides supports that $h \alpha$ silica is a local energy minimum, we further examine the thermodynamic stability by calculating the variation of free energy with temperature. Our results that are derived from the phonon calculations, including the entropy part, indicated in Fig. 1(d) that the system has positive free energy up to $500 \mathrm{~K}$.

To further investigate the effects of temperature on stability, we perform ab initio molecular dynamics (MD) calculations at high temperatures. Since a small unit cell may easily lead to fake instability, we used a relatively large $(4 \times 4)$ supercell. Starting from regular $h \beta$ silica, and by raising the temperature to $1000 \mathrm{~K}$, the structure has transformed to $h \alpha$ silica and did not change in the course of MD simulations lasting $12 \mathrm{ps}$ as shown by the inset of Fig. 1(c). No instability leading to structural transformation or dissociation of $h \alpha$ silica was observed even if the specific phonon modes were softened at such a high temperature. Additionally, through intermediate energy paths, specific atoms were displaced forcibly from their optimized local equilibrium positions corresponding to a minimum in the Born-Oppenheimer surface. The optimized structure was resistant to these deformations and, hence, restored itself quickly. This situation demonstrates that the minimum is

TABLE I. Relevant physical properties of $h \alpha$ silica. $a_{\alpha}$ : hexagonal lattice constant in $\AA$; $d_{1}$ : Si-O bond distance of $s p^{3}$-bonded $\mathrm{Si} ; d_{2}$ : same for $s p^{2}$-bonded $\mathrm{Si}$; $t$ : perpendicular distance between $s p^{3}$-bonded and $s p^{2}$-bonded $\mathrm{Si}$ atoms in $\AA ; E_{c}, E_{f}$ : the cohesive and formation energies per unit cell in eV; $E_{G-\mathrm{GGA}}$ : band gap calculated by generalized gradient approximation (GGA) with van der Waals correction in $\mathrm{eV} ; E_{G-\mathrm{HSE}}$ : band gap calculated by Heyd-Scuseria-Ernzerhof (HSE); $C$ : in-plane stiffness in N/m; $\nu$ : Poisson ratio; $Q_{O}^{*}, Q_{S i-s p^{3}}^{*}, Q_{S i-s p^{2}}^{*}$ : Mulliken charges in electrons for different atoms.

\begin{tabular}{lcccccccccccc}
\hline \hline$a_{\alpha}$ & $d_{1}$ & $d_{2}$ & $\mathrm{t}$ & $E_{c}$ & $E_{f}$ & $E_{G-\mathrm{GGA}}$ & $E_{G-\mathrm{HSE}}$ & $\mathrm{C}$ & $\nu$ & $Q_{O}^{*}$ & $Q_{S i-s p^{3}}^{*}$ & $Q_{S i-s p^{2}}^{*}$ \\
\hline 5.18 & 1.76 & 1.58 & 0.96 & 28.6 & 9.2 & 2.2 & 3.3 & 22.6 & -0.21 & -0.31 & 0.53 & 0.39 \\
\hline \hline
\end{tabular}


deep enough to retain the structure of $h \alpha$ silica stable at ambient conditions.

Our conclusions on the mechanical stability is corroborated further by calculating its in-plane stiffness, $C=$ $A_{o}^{-1} \partial^{2} E_{T} / \partial \epsilon^{2}$ (in terms of the area $A_{o}$ of the cell and the variation of total energy, $E_{T}$ with strain, $\epsilon$ ). Here, calculations can conveniently be performed in a rectangular cell by applying uniaxial strain along $x$ and $y$ directions to deduce that $C_{x} \simeq C_{y}=22.6 \mathrm{~J} / \mathrm{m}^{2}$. The calculated in-plane stiffness is smaller than that of graphene [30].

The Poisson's ratio $\nu=-\epsilon_{y} / \epsilon_{x}$, is calculated as $\nu=-0.21$. This is remarkable in the sense that, as $h \alpha$ silica is stretched along the $x$ direction, it also expands in the $y$ direction, owing to its squeezed structure consisting of twisted and bent $\mathrm{Si}-\mathrm{O}-\mathrm{Si}$ bonds. Thus, three oxygen atoms in each hexagon protrude inward resulting in a reentrant structure [29]. The negative Poisson's ratio is a rare situation [31] and is also observed in foams produced from low density, open-cell polymers [32,33]. These extremal materials, also called auxetics or metamaterials, offer crucial applications for biotechnology and nanosensors.

Electronic properties of $h \alpha$ silica are summarized in Fig. 2, which shows that it is a nonmagnetic, wide band gap semiconductor with a direct band gap of $E_{G-\mathrm{GGA}}=2.2 \mathrm{eV}$ predicted using GGA calculations [23]. When corrected by HSE [24], the gap increases to $E_{G-\mathrm{HSE}}=3.3 \mathrm{eV}$. While conduction and valance band edges are mainly derived from the dangling orbitals of $s p^{2}$-bonded and $s p^{3}$-bonded $\mathrm{Si}$, respectively, the bands of oxygen- $p$ orbitals occur below $\sim-4 \mathrm{eV}$.

According to Mulliken charge analysis [26] summarized in Fig. 2(b), Si-O bonds have a strong ionic character. Because of the special bond configuration, each oxygen atom receives $\sim 0.31$ excess electrons, while 0.53 electrons and 0.39 electrons are donated by $s p^{3}$ - and $s p^{2}$-bonded $\mathrm{Si}$ atoms, respectively. The details of charge transfer are

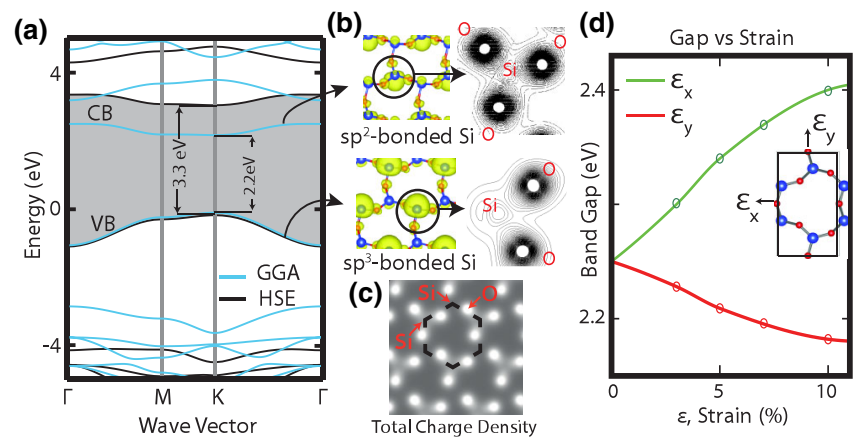

FIG. 2 (color online). (a) The electronic band structure of $h \alpha$ silica. (b) Isosurface charge densities of the lowest conduction (CB) and highest valence (VB) band, and contour plots of the total charge density in the planes of O-Si-O for the $s p^{2}$ - and the $s p^{3}$-bonded $\mathrm{Si}$ atoms. (c) Images of the total charge density. (d) Variation of the band gap as a function of the uniaxial strains $\epsilon_{x}$ and $\epsilon_{y}$. depicted by charge density isosurfaces and contour plots. Charge rearrangements are also reflected by the calculated total charge density in Fig. 2(c), where oxygen and corner $\mathrm{Si}$ atoms are seen by bright and dispersed spots, respectively, while $s p^{3}$-bonded $\mathrm{Si}$ atoms are not seen. Because of the significant charge transfer between $\mathrm{Si}$ and $\mathrm{O}$ atoms, dipole moments along $x$ and $y$ directions are expected to be high.

It is a rather rare situation that the variation of the calculated band gap is strain specific; it increases with increasing uniaxial strain, $\epsilon_{x}$, but it decreases with increasing $\epsilon_{y}$ as shown in Fig. 2(d). Reversed responses of the band gap to the orthogonal uniaxial tensile stresses are closely related to the atomic configuration in the rectangular unit cell described in Fig. 1.

Nanoribbons attribute further relevance to $h \alpha$ silica. In Fig. 3, we consider zigzag and armchair nanoribbons specified by the number of hexagons across their widths $w$, $N_{Z}=4$ and $N_{A}=4$, respectively. Apart from the minute reconstruction at their edges, these nanoribbons are stable. The zigzag $N_{Z}=4$ nanoribbon is a metal, where the flat band at the Fermi level is derived from the orbitals of $s p^{2}$ bonded atoms located at one edge. Another band crossing the Fermi level has small dispersion and originates from the orbitals of $s p^{3}$-bonded $\mathrm{Si}$ atoms at the other edge. In contrast, the armchair nanoribbon is a semiconductor with a direct band gap of $1.9 \mathrm{eV}$ calculated within GGA [23]. The bands at the conduction and valance band edges are derived from the $s p^{2}$-bonded and $s p^{3}$-bonded $\mathrm{Si}$ atoms at both edges, respectively. Because of small coupling between edges, the bands are slightly split. These nanoribbons with or without foreign adatoms attached to the edges and their networks consisting of the combination of zigzag and armchair nanoribbons with different $N_{Z}$ and $N_{A}$, can display a diversity of electronic properties, which is beyond the scope of the present study. Here, we point out a property of $h \alpha$ silica, which may be of potential importance. Because of the significant charge transfer between $\mathrm{Si}$
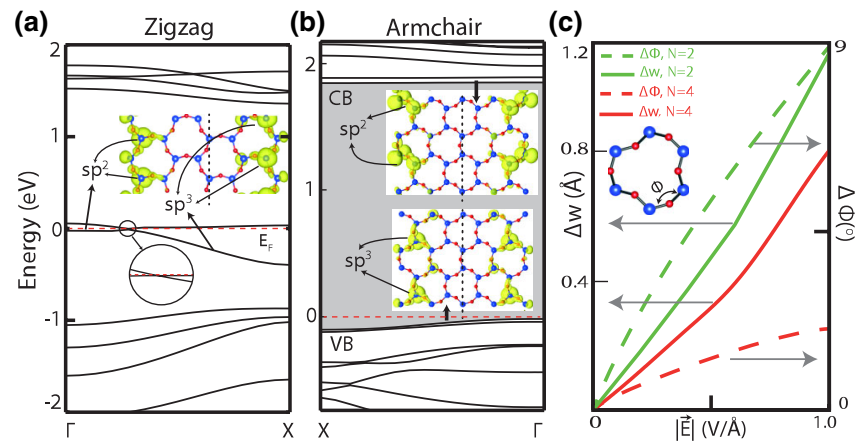

FIG. 3 (color online). (a) Electronic band structure of zigzag nanoribbon with $N_{Z}=4$. (b) Same for the armchair nanoribbon with $N_{A}=4$. (c) Variation of the width of the armchair nanoribbon $\Delta w$ and bond angle $\Delta \phi$ under an in-plane electric field $\vec{E}$ applied perpendicular to the axis. 
(a)

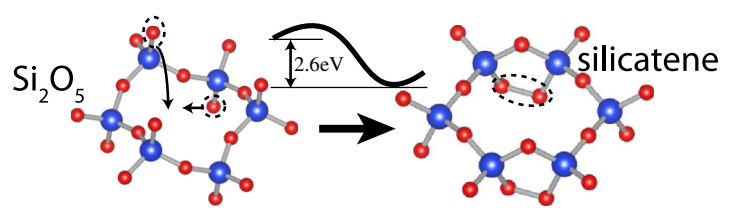

(b)

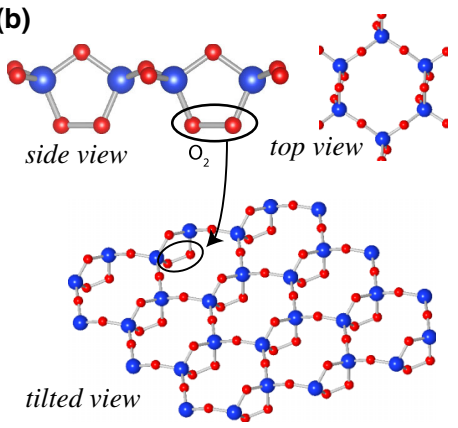

(c)

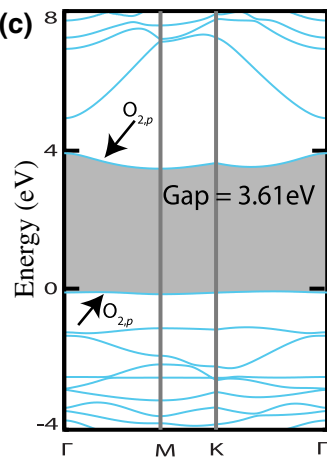

FIG. 4 (color online). (a) Silicatene derived from $\mathrm{Si}_{2} \mathrm{O}_{5}$. (b) Atomic structure of silicatene with side, top, and tilted views. Pentagons are formed from three $\mathrm{O}$ and two Si atoms. (c) Electronic band structure. Bands derived from O-O bonds are indicated.

and $\mathrm{O}$ atoms and its reentrant structure, a finite size flake of $h \alpha$ silica is expected to be influenced by the applied electric field. The effect of the in-plane electric field, $\vec{E}$, is investigated on the armchair nanoribbons. As illustrated in Fig. 3(c), an applied in-plane electric field induces significant changes in the width $\Delta w$ and Si-O-Si bond angle $\phi$ of armchair nanoribbons. The piezoelectric coefficient is estimated from $\Delta w$ as $5.7 \times 10^{-12} \mathrm{~m} / \mathrm{V}$, which is more than twice the value measured for quartz [34], which is crucial for piezoelectric nanodevices.

It should be noted that the basic structure of $h \alpha$ silica and its ribbons discussed so far are reactive due to dangling bonds or $\pi$ orbitals oozing from Si atoms. Thus, they can acquire new functionalities by the adsorption of adatoms to $\mathrm{Si}$, such as $\mathrm{H}, \mathrm{F}, \mathrm{O}$, etc. Like graphene, uniform and full coverage of $h \alpha$ silica by one of these atoms can produce new derivatives $\mathrm{Si}_{2} \mathrm{O}_{3} \mathrm{H}_{2}, \mathrm{Si}_{2} \mathrm{O}_{3} \mathrm{~F}_{2}$, and $\mathrm{Si}_{2} \mathrm{O}_{5}$. A summary of the properties attained by these derivatives, as well as bilayer and multilayer structures are presented in the Supplemental Material [35]. Here, the derivative $\mathrm{Si}_{2} \mathrm{O}_{5}$ is of particular interest. Upon oxidation through the saturation of Si dangling bonds alternating from the top and bottom sides, the filled band at the top of the valance band derived from the orbitals of $s p^{3}$-bonded $\mathrm{Si}$ and the empty band at the bottom of the conduction band derived from the orbitals of $s p^{2}$-bonded $\mathrm{Si}$ atoms in Fig. 2 are removed and the band gap increases from $2.2 \mathrm{eV}$ to $\sim 6 \mathrm{eV}$, attributing a high insulating character and inertness like 3D silica. While the hexagonlike 2D geometry in Fig. 1 is maintained, $s p^{2}$-bonded $\mathrm{Si}$ atoms change to $s p^{3}$-bonded $\mathrm{Si}$ atoms and, hence, restore the rotary reflection symmetry. This way, $\mathrm{Si}$ atoms acquire the fourfold coordination of oxygen atoms in Fig. 4(a) as in 3D silica. However, upon heating, $\mathrm{Si}_{2} \mathrm{O}_{5}$

undergoes a structural transformation by further lowering (i.e., becoming more energetic) its total energy by $2.63 \mathrm{eV}$, whereby the first half of the dangling $\mathrm{Si}-\mathrm{O}$ bonds rotate from top to bottom so that all are relocated at the bottom side. Eventually, they are paired to form $\mathrm{O}-\mathrm{O}$ bonds as shown in Fig. 4(a). The resulting electronic structure of this phase is presented in Fig. 4(b). The filled and empty bands occurring in the band gap of $\mathrm{Si}_{2} \mathrm{O}_{5}$ are derived from O-O bonds. As a final remark, it is gratifying that the optimized atomic structure predicted in Fig. 4(a) replicates the structure of the single layer silica in a honeycomb structure (named silicatene), the growth of which was achieved recently on a $\mathrm{Ru}(0001)$ surface [36]. The measured lattice parameters also agree with the present calculated values. Based on the present analysis, it is revealed that silicatene is bonded to metal substrates through $\mathrm{O}-\mathrm{O}$ bonds below the Si plane.

In conclusion, we showed that the single layer honeycomblike structure of silica, $h \alpha$ silica, is stable and mimics the well-known crystalline quartz in 2D. Additionally, this allotrope and its nanoribbons display exceptional electromechanical properties and can attain bilayer and multilayers through the stacking of silica single layers. While diverse functionalities can be attained by the adsorption of foreign atoms, a new phase named silicatene forms when the dangling $\mathrm{Si}$ orbitals are saturated by oxygen atoms. While the allotropes of bulk silica have made a great impact in fundamental and applied research, the exceptional properties revealed in this Letter herald that $h \alpha$ silica and its derivatives can present potential applications, such as composite materials, functional coatings, and also, as single layer insulators in nanoelectronics and nanocapacitors.

The computational resources have been provided by TUBITAK ULAKBIM, High Performance and Grid Computing Center (TR-Grid e-Infrastructure) and UYBHM at Istanbul Technical University through Grant No. 2-024-2007. This work was partially supported by the Academy of Sciences of Turkey (TUBA). S. Cahangirov acknowledges Marie Curie Grant No. FP7-PEOPLE-2013IEF, project ID 628876, and Grupos Consolidados UPV/ EHU del Gobierno Vasco (Grant No. IT578-13).

*ciraci@fen.bilkent.edu.tr

[1] A. K. Geim and I. V. Grigorieva, Nature (London) 499, 419 (2013).

[2] S. Cahangirov, M. Topsakal, E. Akturk, H. Sahin, and S. Ciraci, Phys. Rev. Lett. 102, 236804 (2009).

[3] The fact that the free standing graphene does exist, but not silicene, cannot be taken as a counter argument against the stability of silicene. All single layer structures, including graphene, need a suitable substrate if they are growing from constituent atoms. Free standing silicene does not exist, 
since there is no 3D allotrope of silicon consisting of weakly bonded layers like graphite, $\mathrm{BN}$, or $\mathrm{MoS}_{2}$

[4] P. Vogt, P. DePadova, C. Quaresima, J. Avila, E. Frantzeskakis, M. C. Asensio, A. Resta, B. Ealet, and G. Le Lay, Phys. Rev. Lett. 108, 155501 (2012).

[5] J. Weissenrieder, S. Kaya, J.-L. Lu, H.-J. Gao, S. Shaikhutdinov, H.-J. Freund, M. Sierka, T. K. Todorova, and J. Sauer, Phys. Rev. Lett. 95, 076103 (2005).

[6] D. Loffer, J. J. Uhlrich, M. Baron, B. Yang, X. Yu, L. Lichtenstein, L. Heinke, C. Buchner, M. Heyde, S. Shaikhutdinov, H. J. Freund, R. Wlodarczyk, M. Sierka, and J. Sauer, Phys. Rev. Lett. 105, 146104 (2010).

[7] P. De Padova, C. Quaresima, B. Olivieri, P. Perfetti, and G. Le Lay, J. Phys. D 44, 312001 (2011).

[8] V. O. Özçelik and S. Ciraci, J. Phys. Chem. C 117, 26305 (2013).

[9] R. Wang, X. Pi, Z. Ni, Y. Liu, S. Lin, M. Xu, and D. Yang, Sci. Rep. 3, 3507 (2013).

[10] A. Molle, C. Grazianetti, D. Chiappe, E. Cinquanta, E. Cianci, G. Tallarida, and M. Fanciulli, Adv. Funct. Mater. 23, 4340 (2013).

[11] P. Y. Huang, S. Kurasch, A. Srivastava, V. Skakalova, J. Kotakoski, A. V. Krasheninnikov, R. Hovden, Q. Mao, J. C. Meyer, J. Smet, D. A. Muller, and U. Kaiser, Nano Lett. 12, 1081 (2012).

[12] T. K. Todorova, M. Sierka, J. Sauer, S. Kaya, J. Weissenrieder, J. L. Lu, H. J. Gao, S. Shaikhutdinov, and H. J. Freund, Phys. Rev. B 73, 165414 (2006).

[13] J. Seifert, D. Blauth, and H. Winter, Phys. Rev. Lett. 103, 017601 (2009).

[14] S. Shaikhutdinov and H-J. Freund, Adv. Mater. 25, 49 (2013).

[15] T. Bjorkman, S. Kurasch, O. Lehtinen, J. Kotakoski, O. V. Yazyev, A. Srivastava, V. Skakalova, J. H. Smet, U. Kaiser, and A. V. Krasheninnikov, Sci. Rep. 3, 3482 (2013).

[16] M. Durandurdu, Phys. Rev. B 81, 174107 (2010).

[17] R. Martonak, D. Danadio, A. R. Oganov, and M. Parinello, Nat. Mater. 5, 623 (2006).

[18] S. Ciraci and I. P. Batra, Phys. Rev. B 15, 4923 (1977).

[19] We performed spin polarized density functional theory calculations within GGA including van der Waals corrections [20,21]. We used projector-augmented wave potentials [22] and the exchange-correlation potential is approximated with the PBE functional [23]. The Brillouin zone was sampled by $(17 \times 17 \times 1) \mathbf{k}$ points in the Monkhorst-Pack scheme. Energy convergence value between two steps was chosen as $10^{-6} \mathrm{eV}$. A maximum force less than $0.001 \mathrm{eV} / \AA$, and pressure less than $0.08 \mathrm{kPa}$ in the unit cell was allowed. Energy band structure calculated by GGA [23] has been corrected using the HSE [24] functional. Phonon dispersions are determined using the small displacement method [25]. Dipole corrections are applied to remove spurious dipole interactions between periodic images. Effective charges on atoms are calculated in terms of atomic orbitals using the SIESTA [26] code. Structure optimizations are repeated using a $(4 \times 4)$ supercell to circumvent the constraints. Ab initio molecular dynamics calculations using the Nose thermostat were performed with a time step of $2.5 \mathrm{fs}$ lasting for $12 \mathrm{ps}$. Atomic velocities were renormalized to the temperatures set at $\mathrm{T}=1000 \mathrm{~K}$ at every 40 time steps.

[20] S. Grimme, J. Comput. Chem. 27, 1787 (2006).

[21] G. Kresse and J. Furthmuller, Phys. Rev. B 54, 11169 (1996).

[22] P. E. Blochl, Phys. Rev. B 50, 17953 (1994).

[23] J. P. Perdew, K. Burke, and M. Ernzerhof, Phys. Rev. Lett. 77, 3865 (1996).

[24] J. Heyd, G. E. Scuseria, and M. Ernzerhof, J. Chem. Phys. 118, 8207 (2003); J. Paier, M. Marsman, K. Hummer, G. Kresse, I. C. Gerber, and J. G. Angyan, J. Chem. Phys. 124, 154709 (2006).

[25] D. Alfe, Comput. Phys. Commun. 180, 2622 (2009).

[26] J. M. Soler, E. Artacho, J. D. Gale, A. Garcia, J. Junquera, P. Ordejon, and D. Sanchez-Portal, J. Phys. Condens. Matter 14, 2745 (2002).

[27] From the symmetry point of view it is equally probable that $s p^{3}$-bonded $\mathrm{Si}$ atoms can be either at the top or bottom side of the plane of oxygen atoms. This implies frustration and may lead to domain structure.

[28] Even though the bond angle of $s p^{3}$-hybrid bonds deviate from tetrahedral angles, we continue to specify related $\mathrm{Si}$ atoms as $s p^{3}$-bonded $\mathrm{Si}$ atoms throughout the text.

[29] The Si-O-Si angles between $s p^{2}$ - and $s p^{3}$-bonded $\mathrm{Si}$ atoms, which are denoted by $\phi$ for oxygens protruding outward and by $\phi^{\prime}$ for oxygen atoms protruding inward, are $140^{\circ}$ and $220^{\circ}$, respectively.

[30] H. Sahin, S. Cahangirov, M. Topsakal, E. Bekaroglu, E. Akturk, R. T. Senger, and S. Ciraci, Phys. Rev. B 80, 155453 (2009).

[31] In Ref. [30], the calculated Poisson's ratio of more than 20 stable, single layer honeycomb structures of Group IV elements and Group III-V compounds were found to be positive.

[32] R. S. Lakes, Science 235, 1038 (1987).

[33] X. F. Wang, T. E. Jones, W. Li, and Y. C. Zhou, Phys. Rev. B 85, 134108 (2012).

[34] V. E. Bottom, J. Appl. Phys. 41, 3941 (1970).

[35] See Supplemental Material at http://link.aps.org/ supplemental/10.1103/PhysRevLett.112.246803 for additional information about bilayer, multilayer, hydrogenated, oxygenated, and fluorinated silica.

[36] B. Yang, J. A. Boscoboinik, X. Yu, S. Shaikhutdinov, and H. J. Freund, Nano Lett. 13, 4422 (2013). 